\title{
Antennas for Wide Area Distributed RFID Systems
}

\author{
Michael Crisp, Rui Chen, Adjeck Ndifon, \\ Richard Penty \\ Electrical Engineering Division, Department \\ of Engineering \\ University of Cambridge \\ Cambridge, UK \\ mjc87@cam.ac.uk
}

\begin{abstract}
This paper describes the use of Wide Area Distributed RFID Systems to enable wide area coverage of UHF RFID with high likelihood of tags anywhere within the area being successfully detected. The implications on antenna design are discussed and novel loop antenna meeting the requirements is presented.
\end{abstract}

Keywords-component, formatting, style, styling, insert (key words)

\section{INTRODUCTION}

RFID technology has been seeing increased commercial interest in recent years resulting in a number of large scale deployments of RFID tags across a wide range of application including apparel retail, asset management and logistics. The majority of these deployments have relied on handheld RFID readers which still require human input to perform a scan of the tagged items, although they have advantages over conventional bar-codes of not requiring line of sight and enabling a higher throughput of items. Fixed RFID readers are still generally limited to applications where tags are constrained to pass close to the RFID reader antennas such as in self checkouts. However, to unlock the full potential of RFID, fixed readers must be deployed over wide areas with reliable tag detection.

The limitation facing fixed RFID readers is the effect of multipath which typically limits the reliable read range (the maximum distance at which a tag can be continuously read while moving away from the reader antenna) to be significantly less than the absolute maximum read range due to the effects of multipath and fading coupled with the relatively narrow frequency allocations which are typically within the channel coherence bandwidth and thus provide little diversity. For a fixed RFID reader deployment with tags which are largely static, tags which fall into multipath nulls, are likely to remain continuously undetected. The limited reliable read range requires very dense antenna deployments which increases the cost of implementation of the RFID even if multiplexers are used to drive a very large number of antennas from a single reader.

This paper reviews techniques using distributed RFID systems where multiple, well separated antennas are used to overcome the effects of fading and achieve a high tag detection accuracy over a wide area. The implications of such systems on antenna design are also discussed.

\section{Distributed RFID WITH PHASE Diversity}

Inbuilding wireless signal distribution for communications suffers from similar challenges to RFID owing to the

This work has been supported by EPSRC under grant EP/S019405/1 highly multipath propagation environment indoors where RFID systems typically operate. A common solution in communications systems to make use of a distributed antenna system where multiple low power antennas simultaneously multi-cast the downlink RF signals [1]. In the case of communications, the relatively widebandwidth of the signals combined with the multi-path resistant modulation formats prevents destructive interference from occurring between the signals transmitted from the multiple antennas. Since the RF propagation losses can be reduced, significant improvements in the RSSI can be achieved over a given area even when the output power of each antenna of the multiple antennas is reduced when compared to a single antenna system [2]. However, if the same system is applied to RFID signals, the narrow bandwidth will result in an interference pattern being generated between all the coherent antennas, which will result in areas of destructive interference which will limit the ability of tags to be powered and read in those areas. Unlike communications systems, continuous (or near continuous) favorable channel conditions are not required for operation in RFID applications - each tag is normally required to only be read infrequently. This realization leads to the concept of phase diversity in distributed RFID systems where multiple antennas transmit simultaneously the same RF signals, but the phase of each antenna is dithered on each attempt (DAS processing), thus altering the physical locations of the constructive and destructive interference [3]. This concept is shown in Figure 1.

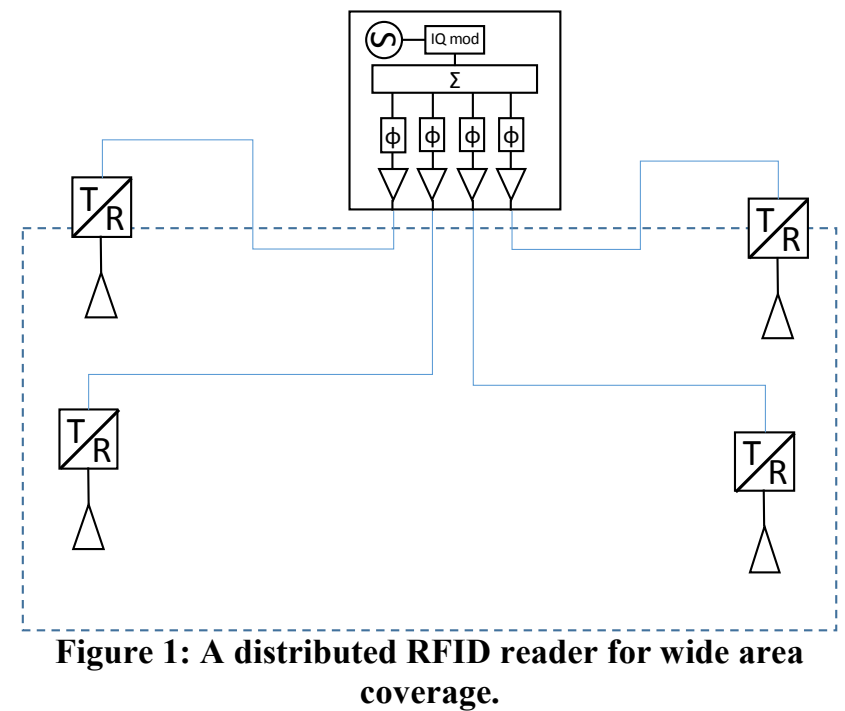

More recently the idea has been extended to show that with a very large number of antennas, the phases can be 
carefully manipulated to create a concentrated hot-spot of RF energy [4] and enable RFID to operate over extreme ranges [5].

\section{ANTENNA DESIGN CONSIDERATIONS}

While the systems outlined in Part II have been demonstrated using conventional off-the-shelf RFID patch antennas, typically having a beam width $<90^{\circ}$ to take advantage of the higher permitted EIRP in both FCC and ETSI regulations for this configuration and circular polarization to cope with RFID tags of unknown orientation, they are not well suited to wide area systems. Multicell distributed RFID deployments will be required in very wide areas, which result in multiple antennas being required to form a sectorized antenna pattern at the vertex of multiple cells [6]. There is also an optimum elevation angle for the antennas to achieve optimum coverage for a particular mounting height, which approaches $90^{\circ}$ as the cell size increases. For many applications, the visual impact of a large number of relatively large patch antennas protruding from the ceiling by a large amount to achieve a high elevation angle in unacceptable, hence there is a desire to produce an antenna which is:

a. Steerable in azimuth through $360^{\circ}$, such that the beam can be directed into any of the cells adjacent to the vertex, thus reducing the number of required antennas

b. Low profile to blend seamlessly and unobtrusively into a ceiling

c. Have a beam pattern which places power at high elevation angles

d. Be circularly polarized with a good axial ratio in the peak beam direction.

e. Have a beam width $<90^{\circ}$

The ability to steer the peak direction of the antenna beam using a phased array antenna has also be shown to further increase the reliable cell size in combination with the phase diversity technique between multiple antennas [7]. This demonstration used a conventional phased array antenna composed of 4 patches and a flexible feed network. Such antennas suffer from a relatively poor axial ratio at high election angles as the direction of optimum axial ratio is on the boresight of the array.

An alternative design which overcomes the axial ratio limitations while meeting the requirements outlined above is shown in figure 2 [8].

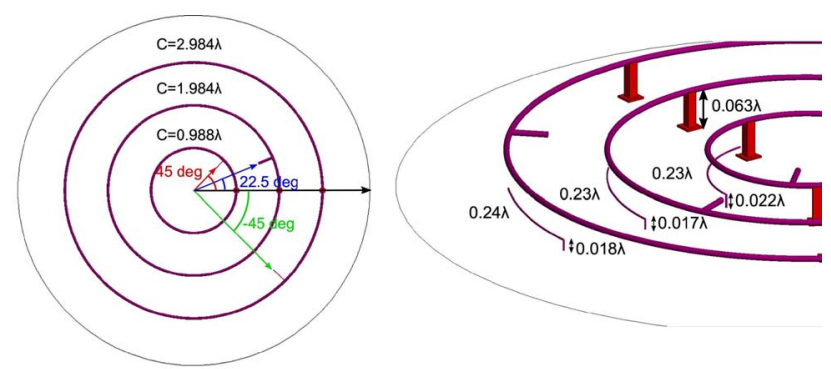

Figure 2: The top and side view of the proposed loop antenna in [8]

It is composed of 3 concentric loops with circumferences increasing by around one wavelength each. A perturbation line is used on each ring to stimulate a travelling wave current distribution and produce circular polarization. The central loop produced a radiation pattern similar to a conventional CP patch, while the outer loops produce conical beam shapes with increasing conic apex angles. By varying the phase of the feeds to each loop, the direction of constructive interference between the patterns can be varied, thereby achieving steering in azimuth; altering the amplitudes of the drives between the loops achieves steering in elevation. As shown in Figure 3, the minimum axial ratio tracks the peak gain as the elevation direction is controlled.

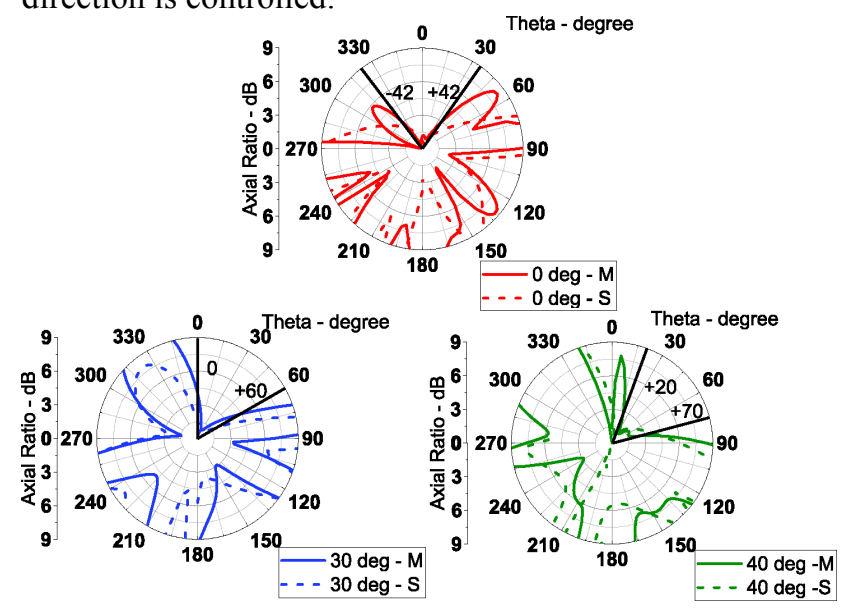

Figure 3: Simulated and measured axial ratio of the proposed antenna against $\theta$ at $\phi=90^{\circ}$ cut, with the peak gain direction at $\theta=0^{\circ}, 30^{\circ}$ and $40^{\circ}$ respectively (M - measured, S - Simulated) [9]

\section{CONCLUSIONS}

This paper has outlined the motivation and potential for very wide area RFID systems, and their requirements in terms of antenna design. A new form of phased array antenna which improves the axial ratio over conventional designs is presented.

\section{REFERENCES}

[1] H. Tang, Lau, K.Y., G. Zhou, and K. Y. Lau, "In-building radio distribution using a "saturated" distributed-antenna-architectureuniversal radio-power and delay-spread statistics," 1999 IEEE International Conference on Communications (Cat. No. 99CH36311), pp. 29-35, Jan 01 1999, doi: 10.1109/ICC.1999.767870.

[2] M. J. Crisp, S. Li, A. Watts, R. V. Penty, and I. H. White, "Uplink and downlink coverage improvements of $802.11 \mathrm{~g}$ signals using a distributed antenna network," Journal of Lightwave Technology, vol. 25, no. 11, pp. 3388-3395, 2007.

[3] S. Sabesan, M. J. Crisp, R. V. Penty, and I. H. White, "Wide area passive UHF RFID system using antenna diversity combined with phase and frequency hopping," IEEE Transactions on Antennas and Propagation, vol. 62, no. 2, pp. 878-888, 2014.

[4] X. Fan et al., "Energy-Ball," Proceedings of the ACM on Interactive, Mobile, Wearable and Ubiquitous Technologies, vol. 2, no. 2, pp. 1-22, 2018, doi: 10.1145/3214268.

[5] J. Wang, J. Zhang, R. Saha, H. Jin, and S. Kumar, "Pushing the range limits of commercial passive RFIDs," in 16th \{USENIX\} Symposium on Networked Systems Design and Implementation (\{NSDI\} 19), 2019, pp. 301-316.

[6] S. Zou, M. Crisp, S. Sabesan, A. Kadri, R. V. Penty, and I. H. White, "An optimization model for antenna selection and deployment in single and multi-cell RFID systems," in RFID Technology and 
Applications (RFID-TA), 2015 IEEE International Conference on, 2015: IEEE, pp. 87-92.

[7] A. M. Ndifon, M. J. Crisp, R. V. Penty, and I. H. White, "Enhanced RFID tag detection accuracy using distributed antenna arrays," presented at the 2018 IEEE International Conference on RFID (RFID), 2018.

[8] R. Chen, S. Yang, A. M. Ndifon, R. V. Penty, I. H. White, and M. J. Crisp, "A UHF RFID reader antenna with tunable axial ratio and fixed beamwidth," in 14th European Conference on Antennas and Propagation (EuCAP), 2020. 